\title{
Measuring co-axial hole size of finite-size metallic disk based on a dual-constraint integration feature using multi-frequency eddy current testing
}

\author{
Ruochen Huang, Mingyang Lu*, Xiaohong He, Anthony Peyton, Wuliang Yin*
}

\begin{abstract}
This paper presents a new approach of eddy current methods for determining the size of the co-axial hole in the metallic circular disk. In recent decades, for the air-cored sensor probe, the impedance change due to the presence of an infinite metal plate can be calculated by the Dodd-Deeds model. However, in practical measurements, the sample cannot match with the condition required - 'infinite', thus the Dodd-Deeds model could not be applied to the disk with finite size and certainly not a co-axial hole in the center. In this paper, a dual-constraint analytical method is proposed. That is, the upper and lower limits of the integration are substituted with specific values instead of the original 0 and $\infty$. Besides, it is found that, once the outer radius of the disk is fixed (i.e. the lower limit of integration is fixed), the upper limit reduces linearly as the size of the coaxial hole increases. Both the FEM simulation and experiments have been carried out to validate this method. The radius of the hole can be estimated based on the dualconstraint integration feature.
\end{abstract}

Index Terms - Hole size measurement, finite-size metallic disk, eddy current testing, non-destructive testing.

\section{INTRODUCTION}

$\mathrm{N}$ on-destructive testing techniques have been applied in the fields of aerospace [1-3], rail transport [4-5], and pipeline testing [6-7]. Due to its advantage of high sensitivity and strong adaptability to the specimen, eddy current testing is widely used for the thickness measurement, liquid level measurement, and defect detection [8-12]. In the eddy current testing, an alternating current is injected into the excitation coil to generate an alternating magnetic field, then the eddy current is induced in the conductive samples. Thus, the receiving coil receives the signal contributed by both the magnetic field from the transmitter and that reflected from the sample.

With the rapid development of computational platforms, the electromagnetic problems can be calculated by numerical analysis algorithms, particularly the finite element method (FEM) $[3,13]$ and the boundary element method (BEM) [14]. FEM is suitable for samples with anisotropic materials but timeconsuming. Ye et al designed an eddy current (EC) probe array

This work was supported by [UK Engineering and Physical Sciences Research Council (EPSRC)] [grant number: EP/P027237/1] [title: Real-time Inline Microstructural Engineering (RIME)].

R. Huang, M. Lu, X. He, A. Peyton and W. Yin are with the School of Electrical and Electronic Engineering, University of Manchester, Sackville with rotating exciting currents for the defect inspection. Besides, the numerical method plays an essential role to validate that the designed probe can detect defects in different layers [15-16].

In recent decades, the Dodd-Deeds analytical method [17-18] has been commonly used for evaluating various eddy current problems, which is more efficient than the numerical approach like FEM. Moulder et al proposed a reliable method to predict the conductivity of the sample and thickness of the substrate metal based on the Dodd-Deeds model [9]. Luloff et al examined Desjardins et al 's model of a non-coaxial sensor probe above two separated sample plates by utilizing the DoddDeeds model. It was found that both methods are matched with the experiments under the low frequency [19]. In [20], by combining the analytical solution and FEM simulations, the ferrite fraction can be inferred with the error typically less than $8 \%$. Moreover, the measurement accuracy also suffers from the effect of the lift-off distance of the sensor. Yin et al. proposed a lift-off immune method to extract the thickness profiles of the samples under different lift-off distance [21]. By incorporating the non-ideal behavior of the eddy current sensor, the modelbased inversion method proposed in [22] proved its reliability in terms of lift-off elimination. Various lift-off compensation algorithms [23-25] have been proposed to eliminate the influence of the lift-off variation in order to predict the material properties more accurately. However, the fundamental formulation of mentioned approaches, i.e. the Dodd-Deeds formulation, cannot be applied for the tested sample with finite dimension, or even with a co-axial hole.

In this paper, to explore the analytical formulation for the finite-size disk sample with a co-axial hole, a dual-constraint integration feature has been found in the Dodd-Deeds model. By introducing two boundary limits, the inductance change due to the presence of the circular sample plate with a co-axial hole can be calculated. In the following sections, the mathematical formulation is presented and validated by the FEM simulation. Furthermore, measurements of the disk sample different sizes

Street Building, Manchester M13 9PL, United Kingdom (Corresponding author: M. Lu, W. Yin, e-mail: mingyang.lu@manchester.ac.uk, $\frac{\text { wuliang.yin@manchester.ac.uk). }}{+}$. 
of the co-axial hole have been carried out. Results show that the size of the co-axial hole can be predicted accurately by utilising the proposed dual-constraint integration feature.

\section{DUAL-CONSTRAINT INTEGRATION FEATURE}

For the EC testing, the impedance change has been acted as a key indicator for the measurement of materials. The change of the impedance due to the sample plate with different kinds of material properties can be computed with various methods. For the air-cored EC sensor, the Dodd-Deeds method has provided the general analytical solution for the circular sensing coil located above the half-space sample plate. Since many test pieces cannot be treated as the infinite sample due to its finite planar size, our modified analytical solution by introducing an initial integration point $\left(\alpha_{r_{s}}\right)$ has been proposed previously to tackle this problem [26].

From our previous research, as shown in Fig. 1, the vector potential generated by the excitation coil placed above the sample plate with finite dimension can be derived as

$$
\mathrm{A}(\mathrm{r}, \mathrm{z})=\frac{\mathrm{I} \mu_{0} \mathrm{~N}_{\mathrm{e}}}{2} \int_{\alpha_{r_{s}}}^{\infty} \frac{\mathrm{J}_{1}(\alpha \mathrm{r}) \mathrm{P}(\alpha)}{\alpha^{3}} \mathrm{~K}(\mathrm{r}, \mathrm{z}, \alpha) \phi(\alpha) \mathrm{d} \alpha
$$

where

$$
\begin{gathered}
\phi(\alpha)=\frac{\left(\alpha_{1}+\mu_{1} \alpha\right)\left(\alpha_{1}-\mu_{1} \alpha\right) \mathrm{e}^{-2 \alpha_{1} \mathrm{D}_{0}}-\left(\alpha_{1}+\mu_{1} \alpha\right)\left(\alpha_{1}-\mu_{1} \alpha\right)}{-\left(\alpha_{1}-\mu_{1} \alpha\right)\left(\alpha_{1}-\mu_{1} \alpha\right) \mathrm{e}^{-2 \alpha_{1} \mathrm{D}_{0}}+\left(\alpha_{1}+\mu_{1} \alpha\right)\left(\alpha_{1}+\mu_{1} \alpha\right)} \\
\alpha_{1}=\sqrt{\alpha^{2}+\mathrm{j} \omega \sigma \mu_{1} \mu_{0}} \\
\mathrm{~K}(\mathrm{r}, \mathrm{z}, \alpha)=2-\mathrm{e}^{-\alpha\left(\mathrm{z}-\mathrm{l}_{\mathrm{e} 2}\right)}-\mathrm{e}^{-\alpha\left(\mathrm{z}-\mathrm{l}_{\mathrm{e} 1}\right)}+\mathrm{e}^{-\alpha \mathrm{z}}\left(\mathrm{e}^{-\alpha \mathrm{l}_{\mathrm{e} 1}}-\mathrm{e}^{\left.-\alpha \mathrm{l}_{\mathrm{e}}\right)}\right) \\
\mathrm{P}(\alpha)=\int_{\alpha \mathrm{r}_{\mathrm{e} 1}}^{\alpha \mathrm{r}_{\mathrm{e} 2}} \tau \mathrm{J}_{1}(\tau) \mathrm{d} \tau
\end{gathered}
$$

Here, I denotes the excitation current, $\mathrm{N}_{\mathrm{e}}$ denotes the turns of the excitation coil, $\mathrm{J}_{1}$ denotes the first-order Bessel function of the first kind, $D_{0}$ denotes the sample thickness, $\mu_{0}$ and $\mu_{1}$ denote the vacuum magnetic permeability and the relative permeability of the sample plate; $\sigma$ denotes the electrical conductivity of the sample plate, $\omega$ denotes the excitation frequency, $\mathrm{l}_{\mathrm{e} 1}$ and $\mathrm{l}_{\mathrm{e} 2}$ denote the lower and upper height of the excitation coil, $r_{\mathrm{e} 1}$ and $\mathrm{r}_{\mathrm{e} 2}$ denote the inner and outer radii of the excitation coil.

As can be seen from equation (2) and (3), $\alpha_{1}$ is a frequencydependent term, the frequency-phase feature of the inductance change due to the presence of the sample plate is mainly dominant by the phase term, $\phi(\alpha)$, which includes sample thickness and conductivity. Besides, other parts are mainly related to the coil geometry and independent of the properties of the sample plate.

According to the differential equation in [2], the magnetic vector potential $\mathbf{A}$ induced in the conductor satisfies,

$$
\frac{\partial^{2} \mathrm{~A}}{\partial \mathrm{r}^{2}}+\frac{1}{\mathrm{r}} \frac{\partial \mathrm{A}}{\partial \mathrm{r}}+\frac{\partial^{2} \mathrm{~A}}{\partial \mathrm{z}^{2}}-\frac{\mathrm{A}}{\mathrm{r}^{2}}+\omega^{2} \mu_{\mathrm{i}} \sigma_{\mathrm{i}} \mathrm{A}-\mathrm{j} \omega \mu_{\mathrm{i}} \sigma_{\mathrm{i}} \mathrm{A}=0
$$

$$
\mathrm{A}=\mathrm{R}(r) Z(z)
$$

Assume that $Z(z)$ satisfies with the condition shown in equation (8),

$$
\frac{1}{\mathrm{Z}(\mathrm{z})} \frac{\partial^{2} \mathrm{Z}(\mathrm{z})}{\partial \mathrm{z}^{2}}=\alpha^{2}-\omega^{2} \mu_{\mathrm{i}} \sigma_{\mathrm{i}}+\mathrm{j} \omega \mu_{\mathrm{i}} \sigma_{\mathrm{i}}
$$

Then combine equations (6) and (7) with equation (8),

$$
\frac{1}{\mathrm{R}(\mathrm{r})} \frac{\partial^{2} \mathrm{R}(\mathrm{r})}{\partial \mathrm{r}^{2}}+\frac{1}{\mathrm{rR}(\mathrm{r})} \frac{\partial \mathrm{R}(\mathrm{r})}{\partial \mathrm{r}}+\alpha^{2}-\frac{1}{\mathrm{r}^{2}}=0
$$

Since $\mathrm{R}(r)$ is a function which is related to the first-order Bessel function of the first kind, equation (9) can be expressed as

$$
(\alpha \mathrm{r})^{2}+\frac{\alpha \mathrm{r}}{4 \mathrm{~J}_{1}(\alpha \mathrm{r})}\left(2\left(\mathrm{~J}_{0}(\alpha \mathrm{r})-\mathrm{J}_{2}(\alpha \mathrm{r})\right)-\alpha \mathrm{r}\left(-3 \mathrm{~J}_{1}(\alpha \mathrm{r})+\mathrm{J}_{3}(\alpha \mathrm{r})\right)\right)=1
$$

As can be seen from equation (10), the product of $\alpha$ and $r$ is the solution of the equation, in consequence, the integration domain of $\alpha$ is inversely proportional to the sample radius, then, the integration domain can be referred to the sample radius.

As shown in Fig. 2, for a metallic plate with finite radius, the lower limit is $\alpha_{r_{s}}$ which corresponds to the position $r=r_{s}$ while the upper limit is $\infty$ which corresponds to the center of the plate $r=0$. Similarly, for the metallic plate with a hole in the center, $\mathrm{r}$ is in the range of $r_{i}$ and $r_{o}$, the integration domain should be from $\alpha_{\mathrm{r}_{o}}$ to $\alpha_{\mathrm{r}_{i}}$.

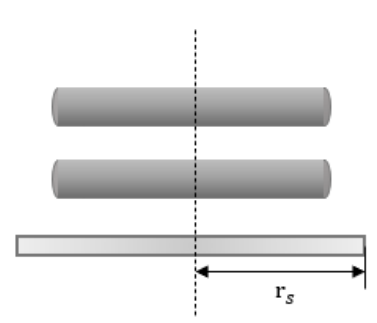

(a)

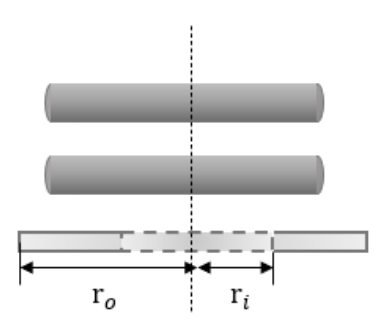

(b)

Fig. 2. EC sensor above the circular sample plate with a finite dimension (a) without the co-axial hole (b) with the co-axial hole

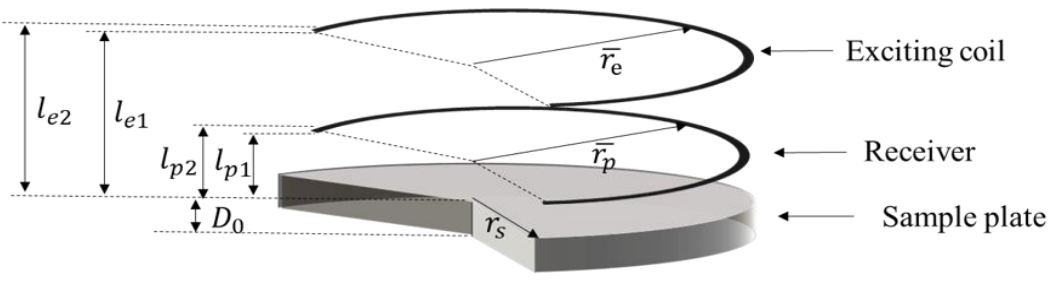

Fig. 1. Illustration of the integrational path for the modified analytical solution 
Thus, as shown in Fig. 3, the magnetic vector potential for the excitation coil above the plate with a co-axial hole in the center is

$$
\begin{aligned}
& \mathrm{A}(\mathrm{r}, \mathrm{z}) \\
& =\frac{\mathrm{I} \mu_{0} \mathrm{~N}_{\mathrm{e}}}{2} \int_{\alpha_{r_{o}}}^{\alpha_{r_{i}}} \frac{\mathrm{J}_{1}(\alpha \mathrm{r}) \mathrm{P}(\alpha)}{\alpha^{3}} \mathrm{~K}(\mathrm{r}, \mathrm{z}, \alpha) \phi(\alpha) \mathrm{d} \alpha
\end{aligned}
$$

Then the induced voltage on the receiver and the impedance change due to the sample plate are calculated as,

$$
\begin{aligned}
& \mathrm{V}=\mathrm{j} \omega \mathrm{N}_{p} \int_{\mathrm{S}} \mathrm{Ads}= \\
& \frac{\mathrm{j} 2 \pi \omega \bar{r}_{\mathrm{p}} \mathrm{N}_{\mathrm{p}}}{\left(\mathrm{l}_{\mathrm{p} 1}-\mathrm{l}_{\mathrm{p} 2}\right)^{2}\left(\mathrm{r}_{\mathrm{p} 1}-\mathrm{r}_{\mathrm{p} 2}\right)^{2}} \int_{\mathrm{l}_{\mathrm{p} 1}}^{\mathrm{l}_{\mathrm{p} 2}} \int_{\mathrm{r}_{\mathrm{p} 1}}^{\mathrm{r}_{\mathrm{p} 2}} \mathrm{rA}(\mathrm{r}, \mathrm{z}) \mathrm{drdz} \\
& \Delta \mathrm{Z}(\omega)=\frac{\Delta V}{\mathrm{I}} \\
& =\frac{\mathrm{j} \omega \pi \bar{r}_{p} \mathrm{~N}_{\mathrm{p}} \mathrm{N}_{\mathrm{e}} \mu_{0}}{\left(\mathrm{l}_{\mathrm{p} 1}-\mathrm{l}_{\mathrm{p} 2}\right)^{2}\left(\mathrm{r}_{\mathrm{p} 1}-\mathrm{r}_{\mathrm{p} 2}\right)} \int_{\alpha_{r_{o}}}^{\alpha_{r_{i}}} \frac{\mathrm{P}^{2}(\alpha)}{\alpha^{6}} \mathrm{e}^{-\alpha\left(2 l+\mathrm{l}_{\mathrm{e} 2}-\mathrm{l}_{\mathrm{e} 1}+\mathrm{g}\right)}(1 \\
& \left.-\mathrm{e}^{-2 \alpha\left(\mathrm{l}_{\mathrm{e} 2}-\mathrm{l}_{\mathrm{e} 1}\right)}\right) \phi(\alpha) \mathrm{d} \alpha \\
& \text { with } \alpha_{r_{x}}=\frac{3.518}{r_{x}} .
\end{aligned}
$$

where: $\mathrm{x}$ can be $i$ or $o$ (the inner or outer radius of the sample plate).

The inductance change due to the sample plate should be

$$
\begin{aligned}
& \Delta \mathrm{L}(\omega)=\frac{\Delta Z}{\mathrm{j} \omega} \\
& =\frac{\pi \bar{r} \mathrm{~N}_{\mathrm{p}} \mathrm{N}_{\mathrm{e}} \mu_{0}}{\left(\mathrm{l}_{\mathrm{p} 1}-\mathrm{l}_{\mathrm{p} 2}\right)^{2}\left(\mathrm{r}_{\mathrm{p} 1}-\mathrm{r}_{\mathrm{p} 2}\right)} \int_{\alpha_{r_{o}}}^{\alpha_{r_{i}}} \frac{\mathrm{P}^{2}(\alpha)}{\alpha^{6}} \mathrm{e}^{-\alpha\left(2 l+\mathrm{l}_{\mathrm{e} 2}-\mathrm{l}_{\mathrm{e} 1}+\mathrm{g}\right)}(1 \\
& \left.-\mathrm{e}^{-2 \alpha\left(\mathrm{l}_{\mathrm{e} 2}-\mathrm{l}_{\mathrm{e} 1}\right)}\right) \phi(\alpha) \mathrm{d} \alpha
\end{aligned}
$$

where: $\mathrm{N}_{\mathrm{p}}$ denotes the turns of the receiving coil, $\overline{r_{p}}$ denotes the average radius of the receiver, $\mathrm{l}_{\mathrm{p} 1}$ and $\mathrm{l}_{\mathrm{p} 2}$ denote the lower and upper height of the receiver, $r_{\mathrm{p} 1}$ and $\mathrm{r}_{\mathrm{p} 2}$ denote the inner and outer radii of the receiver, $l$ denotes the lift-off of the sensor probe, $g$ denotes the gap between the transmitter and receiver, $r_{i}$ denotes the radius of the co-axial hole and $r_{o}$ denotes the radius of the sample plate.

\section{NUMERICAL VERIFICATION}

\section{A. FEM modelling}

In order to validate the proposed method, FEM simulations have been carried out in commercial software - Comsol. In the simulation, the exciter coil, the receiver coil, and the sample plate are co-axially deployed. The sensor parameter is listed in
Table I. The inner and outer radii of the sensor coil were set to 28 and $28.25 \mathrm{~mm}$ respectively. The gap between the exciter coil and the receiver coil was set to $5 \mathrm{~mm}$. The electrical conductivity and the relative magnetic permeability of the sample plate were kept to that of the copper - $59 \mathrm{MS} / \mathrm{m}$ and 1 respectively. The current with the amplitude of $1 \mathrm{~A}$ was injected into the exciting coil while the induced field can be detected by the receiver coil. Three cases have been simulated to test the feasibility of the method. The models of the sample plate with a thickness of $1 \mathrm{~mm}$ are shown in Fig. 4.

TABLE I

SENSOR PARAMETERS

\begin{tabular}{ccc}
\hline \hline The exciter coil & Inner radius $\left(\mathrm{r}_{\mathrm{e} 1}\right)$ & $28 \mathrm{~mm}$ \\
& Outer radius $\left(\mathrm{r}_{\mathrm{e} 2}\right)$ & \\
& & $28.25 \mathrm{~mm}$ \\
\hline The receiver coil & Inner radius $\left(\mathrm{r}_{\mathrm{p} 1}\right)$ & $28 \mathrm{~mm}$ \\
& Outer radius $\left(\mathrm{r}_{\mathrm{p} 2}\right)$ & $28.25 \mathrm{~mm}$ \\
& The exciter coil & 30 \\
\hline Number of turns & The receiver coil & 30 \\
\hline Height of the exciter coil $\left(\mathrm{l}_{\mathrm{e} 2}-\mathrm{l}_{\mathrm{e} 1}\right)$ & $8 \mathrm{~mm}$ \\
Height of the receiver coil $\left(\mathrm{l}_{\mathrm{p} 2}-\mathrm{l}_{\mathrm{p} 1}\right)$ & $8 \mathrm{~mm}$ \\
The gap between two coils $(\mathrm{g})$ & $5 \mathrm{~mm}$ \\
Plate thickness $\left(\mathrm{D}_{0}\right)$ & $1 \mathrm{~mm}$ \\
\hline
\end{tabular}

As can be seen from Fig. 4, the sample plate with a radius of $50 \mathrm{~mm}$ is firstly simulated, then the second step is to simulate the large plate with a small co-axial hole inside. By combining these two features, the inductance change due to the presence of the finite-sized sample plate with a co-axial hole can be calculated by using the analytical method in (14).

\section{B. Analyzing}

1). The finite sample plate (r: $0-50 \mathrm{~mm})$

Figure 5 depicts the real part and the imaginary part of the inductance change using the analytical and numerical FEM method. Compared with the results obtained from the simulation, the proposed method can demonstrate the inductance change well which is perfectly harmonious with the numerical FEM results.

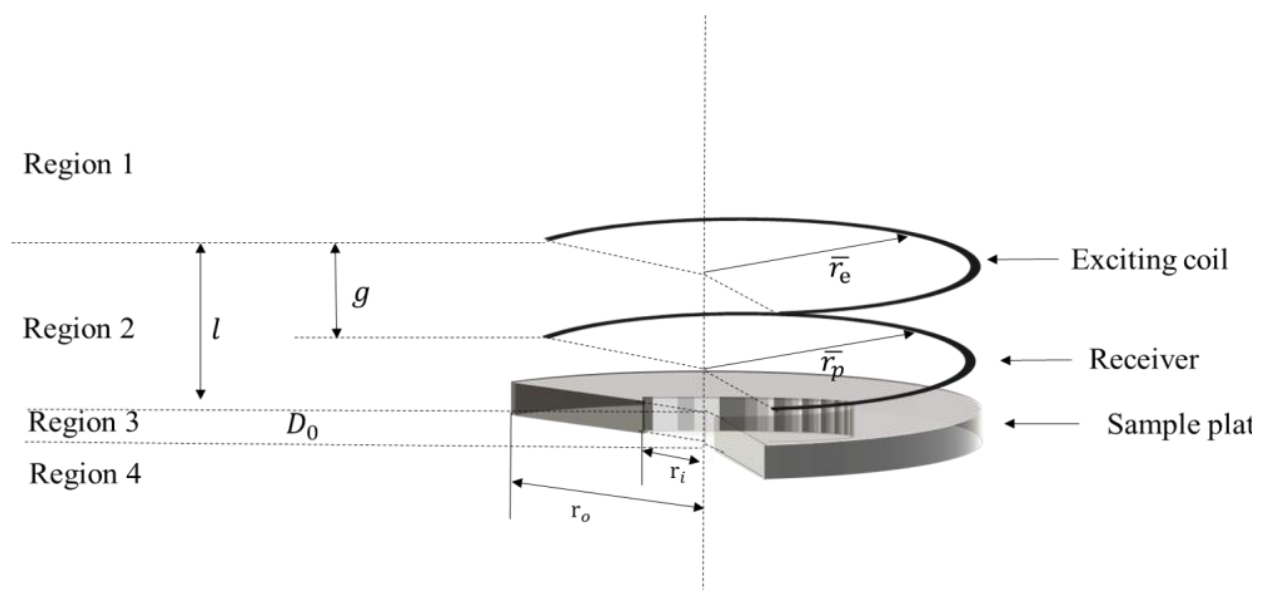

Fig.3 EC sensor above the finite-sized metallic plate with a co-axial hole 


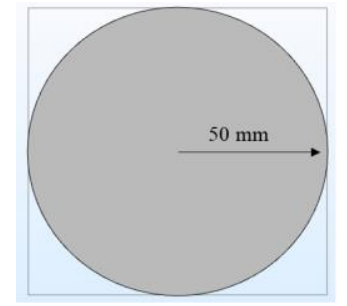

(a)

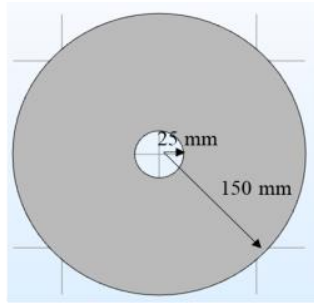

(b)

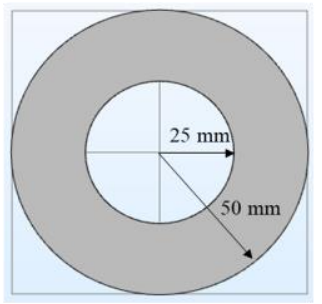

(c)

The proposed method with the aid of Dodd and Deeds model

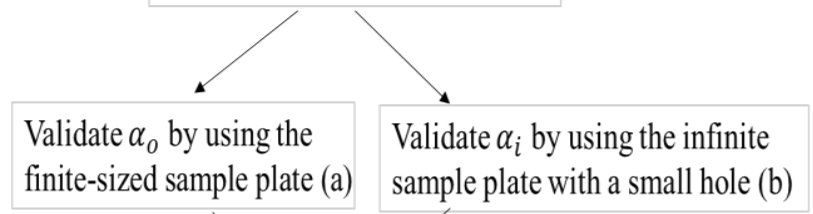

(d)

Fig. 4. (a) finite-size sample plate (b) infinite-size (extremely large) sample plate with a hole (c) finite-size plate with a co-axial hole (d) the flowchart for the validation of the proposed method

As can be seen from Fig. 5(a), there is no zero-crossing point in the real part of the inductance change because of the nonmagnetic metallic sample plate. Besides, under the low frequency, the real part of the inductance change is close to zero. Then, as the frequency increases, the inductance increases gradually until reaching its saturated value. It almost remains stable under the high frequency. Meanwhile, as shown in Fig. 5 (b), the imaginary part of the inductance change is nearly zero under low and high frequencies. However, it can be observed that a peak value exists. From our previous studies, the corresponding frequency - termed as the peak frequency feature, is determined by the properties of the sample plate.

2). The infinite sample plate with a co-axial hole (r: $25 \mathrm{~mm}$ inf)

The inductance due to the hole of a sample with an extremely large planar size is plotted in Fig.6. As shown in Fig. 6, by introducing the upper limit of the integration domain, the method holds for the coil placed above the infinitely large (halfspace) sample plate with a hole. Besides, there is a good match of the inductance change between the proposed method and the numerical FEM simulation. It can be observed that the magnitude of the imaginary part reduces (compared to Fig. 5)

with the peak frequency increased from approximately $200 \mathrm{~Hz}$ to $300 \mathrm{~Hz}$.

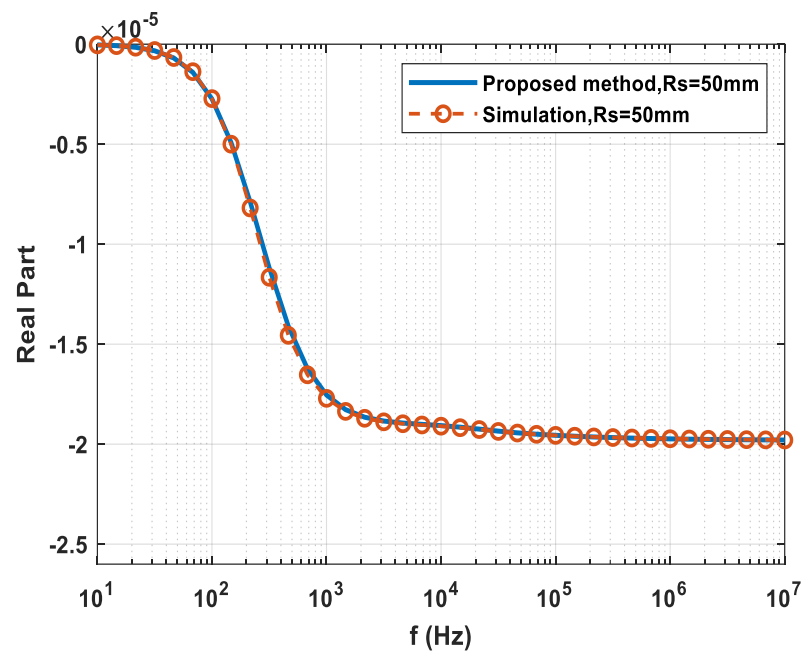

(a)

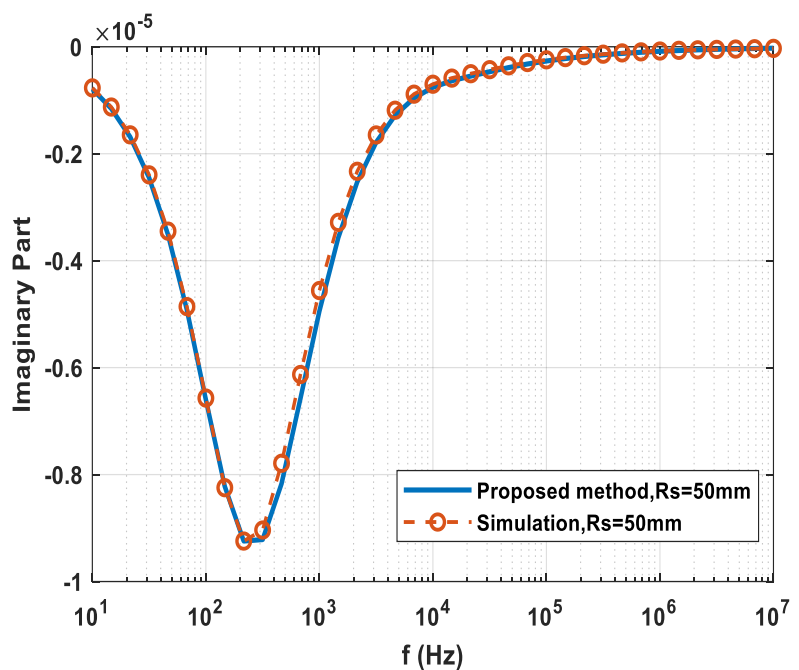

(b)

Fig. 5 Inductance change due to the presence of the small disk sample plate (a) real part (b) imaginary part

3). The finite disk sample plate with a co-axial hole (r: $25 \mathrm{~mm}$ - $50 \mathrm{~mm}$ )

Figure 7 describes the change of the real part and imaginary part of the inductance due to the presence of the finite-size circular sample plate with a co-axial hole. Combining two boundary limits, the inductance change can be well described by the proposed analytical method. There is a slight discrepancy near the frequency $1 \mathrm{kHz}$. It is may because the discretization of the mesh domain in the modelling. Due to the skin depth effect, a much denser mesh is needed for the FEM modelling. Besides, the mesh domain of the free space region could affect the accuracy.

More combinations have been simulated to verify the method in Fig. 8. Moreover, it is found that the upper/lower limit of $\alpha$ $\left(\alpha_{r_{i}} / \alpha_{r_{o}}\right)$ for the analytical formula is proportional to the reciprocal of the inner/outer radius of the sample $\left(r_{i} / r_{o}\right)$. That is, $\alpha_{r_{i}}=\frac{3.518}{r_{i}}$ and $\alpha_{r_{o}}=\frac{3.518}{r_{o}}$, which fits the manipulated formulation in equation (13)). 


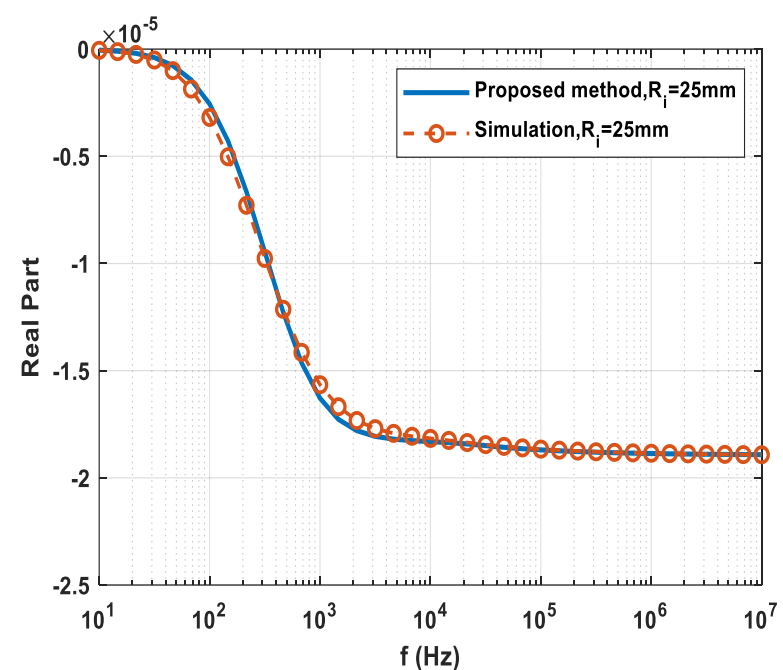

(a)

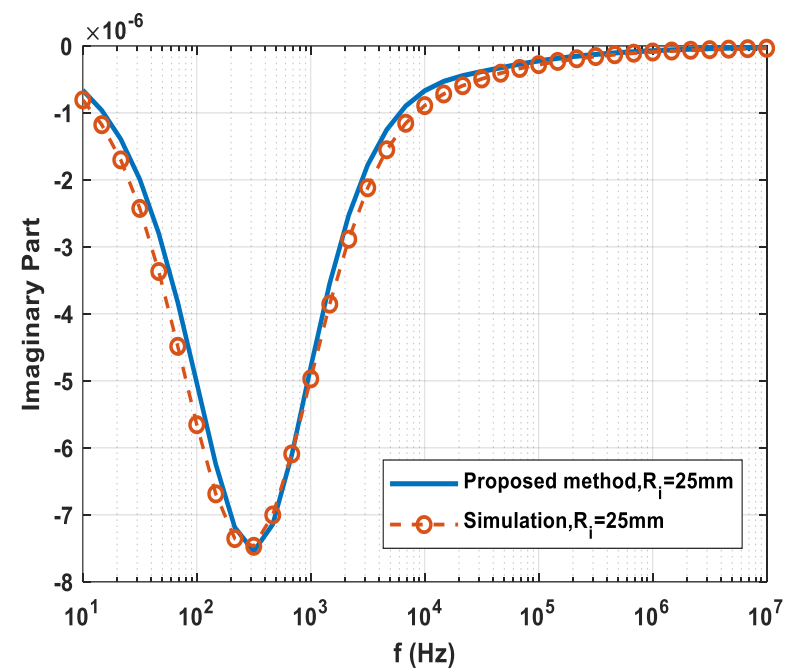

(b)

Fig. 6 Inductance change due to the presence of the half-space sample plate with a small hole (a) real part (b) imaginary part

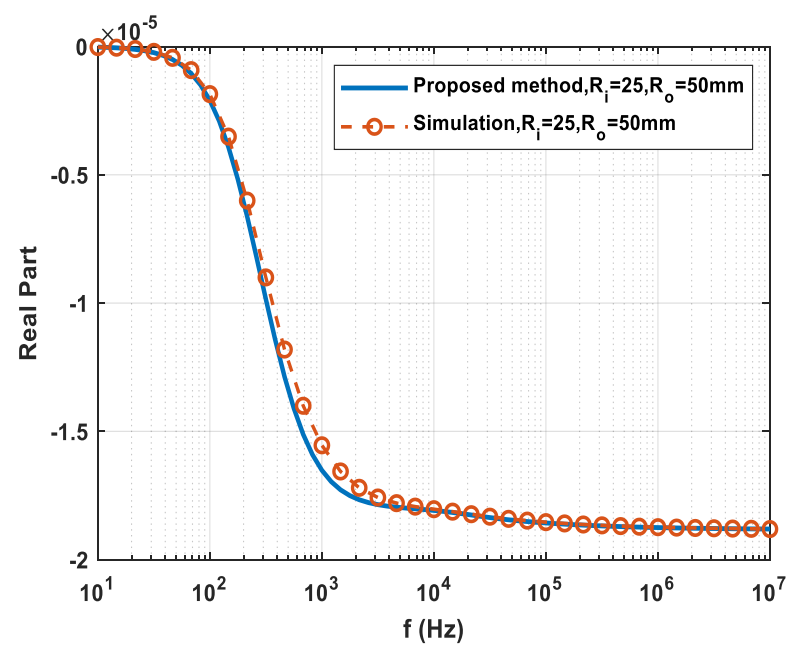

(a)

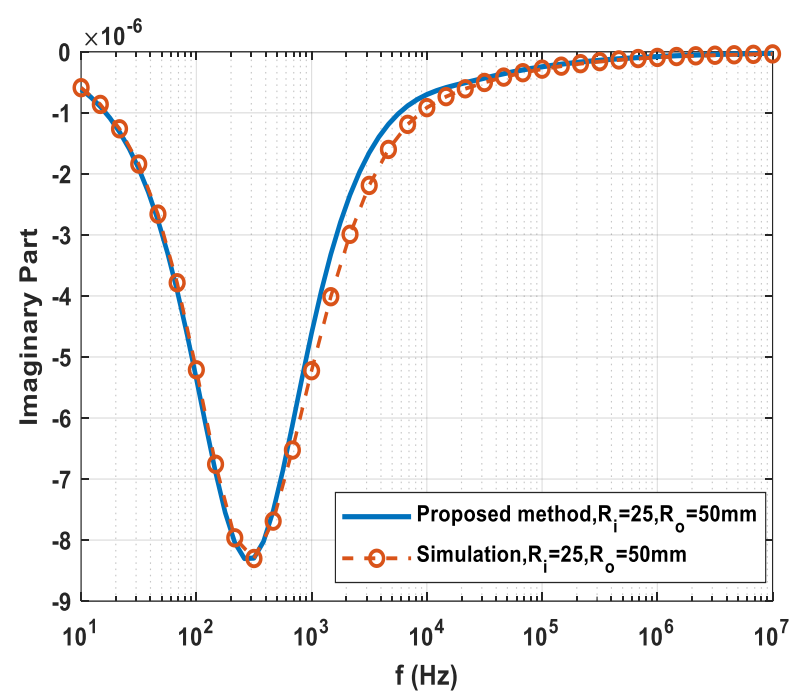

(b)

Fig. 7 Inductance change due to the presence of the finite-size disk sample plate with a co-axial hole (a) real part (b) imaginary part
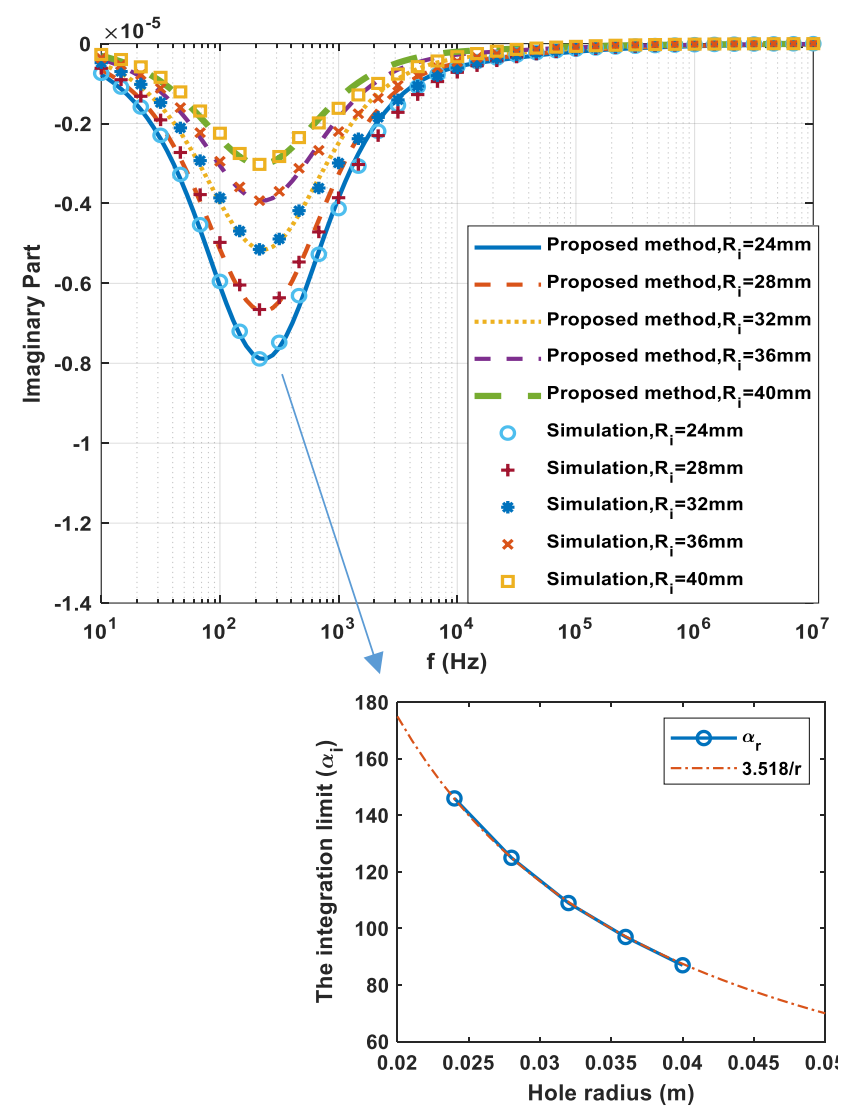

Fig. 8 Imaginary part of the inductance change with the sample plate of different inner radii (i.e. radii of the co-axial hole) and same outer radii

\section{HOLE SIZE MEASUREMENTS}

\section{A. Experimental setup}

Experimental studies have also been conducted and used for the radius measurement of the co-axial hole in the disk sample plate. Fig. 9 shows the entire setup of the experiment system. The probe was situated co-axially to the specimen with different 
radii of the holes. Parameters of the sensor are listed in Table I. The radii of the holes are 12.5, 15, 17.5, and $20 \mathrm{~mm}$ respectively. The material of the sample plate is copper and the thickness is $20 \mu \mathrm{m}$. The impedance analyser was running under the swept frequency mode. Hence, the inductance between the exciting coil and the receiver coil with and without the sample plate can be obtained.

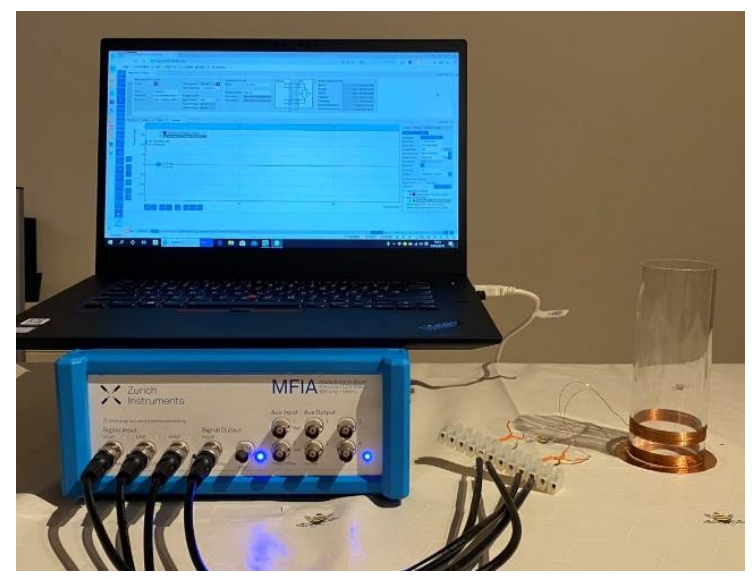

Fig. 9 Experiment system

\section{B. Hole size measurements}

Figure 10 demonstrates the measurement results of the inductance change of the EC sensor above the circular sample plate with various radii of co-axial holes. It can be seen that, as the radius of the co-axial hole increases, the magnitude of the imaginary inductance reduces with the increased peak frequency. The size of the co-axial hole can be predicted by utilising the peak frequency feature. It can be noticed that, as the peak frequency in Fig. $10(20 \mu \mathrm{m}$ case $)$ is much larger than that in Fig. 8 (1 $\mathrm{mm}$ case), the eddy current for the peakfrequency in Fig. 10 is more restraint to the surface of the sample plate. Consequently, the eddy current density under the peak-frequency in Fig. 10 is larger than that in Fig. 8. Thus, the frequency feature becomes more sensitive to the diameter of the sample.

From the experimental data, the peak frequency for each sample plate can be obtained by finding the minimum value of the imaginary part of the inductance. Then we fit the measured peak frequency with the simulations by searching for the upper limit - $\alpha_{r_{i}}$ in (14) (e.g. sweeping the limit from 1000 to 100 in a certain increment, i.e. 1). Further, the relationship between the upper limit $-\alpha_{r_{i}}$ and the radius of the co-axial hole $r_{i}$ is used for the size prediction. Table II lists the actual and measured size by the air-cored EM sensor. The error between the actual and measured radius is within $5 \%$. The difference between the actual and predicted values is due to the approximation of the relationship in (13) and the error caused by the measured peak frequency of the imaginary inductance change.

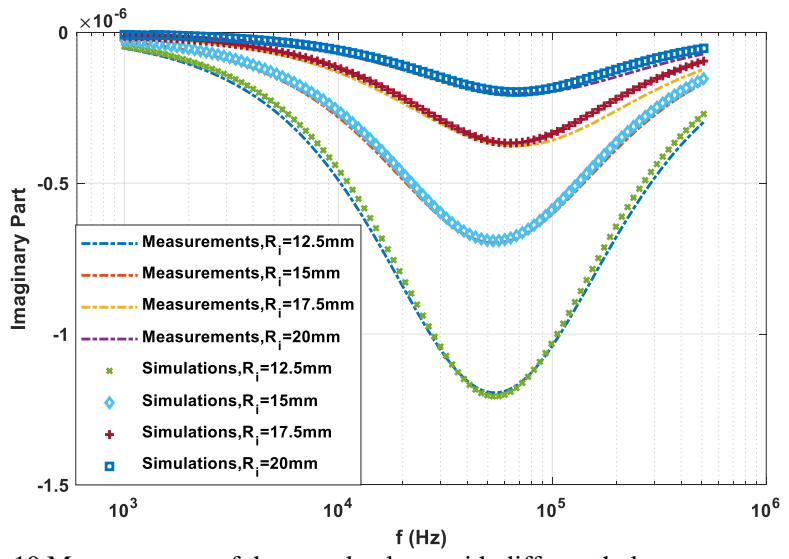

Fig. 10 Measurements of the sample plates with different holes

TABLE II

ACTUAL AND MEASURED RADIUS FOR THE COPPER PLATES

\begin{tabular}{cccc}
\hline \hline Material & $\begin{array}{c}\text { Actual radius of } \\
\text { hole }(\mathrm{mm})\end{array}$ & $\begin{array}{c}\text { Measured radius } \\
\text { of hole }(\mathrm{mm})\end{array}$ & Error $(\%)$ \\
\hline Copper & 12.5 & 12.3 & 1.25 \\
& 15 & 15.3 & -1.97 \\
& 17.5 & 17.1 & 2.41 \\
& 20 & 19.2 & 4.00 \\
\hline
\end{tabular}

\section{CONCLUSIONS}

In the paper, the analytical solution for the impedance of a sensor above the circular disk sample with a co-axial hole has been proposed, which has introduced a dual-constraint integration feature. The dual constraint of the integration has been found inversely proportional to the radii of disk samples and co-axial holes. This method was validated by both the numerical FEM modelling and experiments. By referring to the inversely proportional relationship between the upper constraint, the radius of the hole can be derived. The error for the radius measurement of the co-axial hole can be controlled within $5 \%$. The reconstruction error may be due to the approximated peak frequency from the measurements and the offset of the experimental system.

\section{ACKNOWLEDGEMENT}

This work was supported by [UK Engineering and Physical Sciences Research Council (EPSRC)] [grant number: EP/P027237/1] [title: Real-time In-line Microstructural Engineering (RIME)].

\section{REFERENCES}

[1]. Y. He, F. Luo, M. Pan, F. Weng, et al, "Pulsed eddy current technique for defect detection in aircraft riveted structures," NDT \& E International, vol. 43, no. 2, pp. 176 - 181, 2010. 
[2]. N. V. Nair, V. R. Melapudi, H.R. Jimenez, X. Liu et al., "A GMRBased Eddy Current System for NDE of Aircraft Structures," in IEEE Transactions on Magnetics, vol. 42, no. 10, pp. 3312-3314, Oct. 2006.

[3]. Z. Zeng et al., "A Parametric Study of Magneto-Optic Imaging Using Finite-Element Analysis Applied to Aircraft Rivet Site Inspection," IEEE Transactions on Magnetics, vol. 42, no. 11, pp. 3737-3744, Nov. 2006.

[4]. M.Ph. Papaelias, M.C. Lugg, C. Roberts, C.L. Davis, "High-speed inspection of rails using ACFM techniques," NDT \& E International, vol. 42 , no. 4, pp. $328-335,2009$.

[5]. T. Heckel, H. M. Thomas, M. Kreutzbruck, and S. Rühe, "High Speed Non-Destructive Rail Testing with Advanced Ultrasound and EddyCurrent Testing Techniques," NDTIP Proceedings, Prague, 2009.

[6]. A. A. Carvalho, J. M. A. Rebello, M. P. V. Souza, et al. "Reliability of non-destructive test techniques in the inspection of pipelines used in the oil industry," International journal of pressure vessels and piping, vol. 85, no. 11, pp. 745-751, 2008.

[7]. Z. Xu, X. Wu, J. Li, Y. Kang, "Assessment of wall thinning in insulated ferromagnetic pipes using the time-to-peak of differential pulsed eddycurrent testing signals," NDT \& E International, vol. 51, pp. 24-29, 2012.

[8]. T. Yang, Q. Zhao, K. Y. How, K. Xu, et al, 'Level measurement for saline with a small surface area using high frequency electromagnetic sensing technique,' Measurement, vol. 101, pp. 118-125. 2017.

[9]. J. C. Moulder, E. Uzal and J. H. Rose. "Thickness and conductivity of metallic layers from eddy current measurements," Review of scientific instruments, vol. 63, no. 6, pp. 3455-3465, 1992.

[10]. J. Král, R. Smid, H. M. G. Ramos and A. L. Ribeiro, "Thickness measurement using transient eddy current techniques," 2011 IEEE International Instrumentation and Measurement Technology Conference, Binjiang, pp. 1-6, 2011.

[11]. G. Y. Tian and A. Sophian, "Defect classification using a new feature for pulsed eddy current sensors," Ndt \& E International, vol. 38, no. 1, pp. 77-82, 2005.

[12]. F. Lucklum and B. Jakoby, "Non-contact liquid level measurement with electromagnetic-acoustic resonator sensors," Measurement Science and Technology, vol. 20, no. 12, ,2009.

[13]. N. Moes, J. Dolbow and T. Belytschko, "A Finite Element Method for Crack Growth without Remeshing", International Journal for Numerical Methods in Engineering, vol. 46, pp. 131-150, 1999.

[14]. J. Smajic, Z. Andjelic and M. Bebendorf, "Fast BEM for Eddy-Current Problems Using H-Matrices and Adaptive Cross Approximation," IEEE Transactions on Magnetics, vol. 43, no. 4, pp. 1269-1272, Apr. 2007.

[15]. C. Ye, A. Rosell, M. Haq, et al., "EC probe with orthogonal excitation coils and TMR sensor for CFRP inspection," International Journal of Applied Electromagnetics and Mechanics, vol. 59, no. 4, pp. 12471255, 2019.

[16]. C. Ye, Y. Huang, L. Udpa and S. S. Udpa, "Differential sensor measurement with rotating current excitation for evaluating multilayer structures," IEEE Sensors Journal, vol. 16, pp. 782-789, 2015.

[17]. C. V. Dodd, and W. E. Deeds. "Analytical solutions to eddy-current probe-coil problems." Journal of applied physics, vol. 39, no. 6, pp. 2829-2838, 1968.

[18]. C. V. Dodd, W. E. Deeds, J. W. Luquire and W. G. Spoeri, "Some Eddy-Current Problems and Their Integral Solutions", Oak Ridge National Laboratory, April 1969.

[19]. M. S. Luloff, J. Morelli, T. W. Krause, "Examination of Dodd and Deeds solutions for a transmit-receive eddy current probe above a layered planar structure," AIP Conference Proceedings. AIP Publishing LLC, vol. 1806, no. 1, 2017.

[20]. W. Yin, X. J. Hao, A. Peyton, et al., "Measurement of permeability and ferrite/austenite phase fraction using a multi-frequency electromagnetic sensor,” NDT \& e International, vol. 42, no. 1, pp. 6-68, 2009.

[21]. W. Yin and K. Xu, "A Novel Triple-Coil Electromagnetic Sensor for Thickness Measurement Immune to Lift-Off Variations," in IEEE Transactions on Instrumentation and Measurement, vol. 65, no. 1, pp. 164-169, Jan. 2016.
[22]. M. Fan, B. Cao, P. Yang, et al., "Elimination of liftoff effect using a model-based method for eddy current characterization of a plate," NDT \& E International, vol. 74, pp. 66-71, 2015.

[23]. M. Lu, W. Zhu, L. Yin, A. J. Peyton, W. Yin and Z. Qu, "Reducing the Lift-Off Effect on Permeability Measurement for Magnetic Plates from Multifrequency Induction Data," in IEEE Transactions on Instrumentation and Measurement, vol. 67, no. 1, pp. 167-174, Jan. 2018.

[24]. M. Lu, L. Yin, A. J. Peyton and W. Yin, "A Novel Compensation Algorithm for Thickness Measurement Immune to Lift-Off Variations Using Eddy Current Method," in IEEE Transactions on Instrumentation and Measurement, vol. 65, no. 12, pp. 2773-2779, Dec. 2016.

[25]. M. Lu, R. Huang, W. Yin, Q. Zhao and A. Peyton, "Measurement of Permeability for Ferrous Metallic Plates Using a Novel Lift-Off Compensation Technique on Phase Signature," in IEEE Sensors Journal, vol. 19, no. 17, pp. 7440-7446, 1 Sept.1, 2019.

[26]. R. Huang, M. Lu, A. Peyton, and W. Yin, "Thickness measurement of metallic plates with finite planar dimension using eddy current method," IEEE Transactions on Instrumentation and Measurement, vol. 69, no. 10, 2020. Doi:10.1109/TIM.2020.2987413.

[27]. R. Huang, M. Lu et al. "A novel perturbed matrix inversion based method for the acceleration of finite element analysis in crack-scanning eddy current NDT," IEEE Access, vol. 8, pp. 12438-12444, 2020.

[28]. W. Yin, M. Lu et al. "Custom edge-element FEM solver and its application to eddy-current simulation of realistic $2 \mathrm{M}$-element human brain phantom," Bioelectromagnetics, vol. 39, pp. 604-616, 2018.

[29]. W. Yin, M. Lu et al. "Acceleration of eddy current computation for scanning probes," Insight-Non-Destructive Testing and Condition Monitoring, vol. 60, pp. 547-555, 2018.

[30]. J. Tang et al. "A Novel Efficient FEM Thin Shell Model for BioImpedance Analysis," Biosensors, vol. 10, pp. 69, 2020.

[31]. Y. Xie, M. Lu et al. "Novel Wearable Sensors for Biomechanical Movement Monitoring Based on Electromagnetic Sensing Techniques," IEEE Sensors J., vol. 20, no. 2, pp. 1019-1027, 2019.

[32]. M. Lu et al. "Forward solver for deep earth exploration and induction logging using custom built Edge-Element FEM technique," Acta Geologica Sinica - English Edition, vol. 93, pp. 302-304, 2019.

[33]. M. Lu, H. Xu, W. Zhu, L. Yin et al. "Conductivity Lift-off Invariance and measurement of permeability for ferrite metallic plates," NDT \& E International, vol. 95, pp. 36-44, Apr. 2018.

[34]. M. Lu, R. Huang, W. Yin, Q. Zhao, and A. Peyton, "Measurement of permeability for ferrous metallic plates using a novel lift-off compensation technique on phase signature," IEEE Sensors Journal, vol. 19, no. 17, pp. 7440-7446, 1 Sept.1, 2019.

[35]. M. Lu, et al. "Determination of the magnetic permeability, electrical conductivity, and thickness of ferrite metallic plates using a multifrequency electromagnetic sensing system," IEEE Transactions on Industrial Informatics, vol. 15, pp. 4111-4119, 2019.

[36]. M. Lu, et al. "Acceleration of frequency sweeping in eddy-current computation," IEEE Transactions on Magnetics, vol. 53, pp. 1-8, 2017.

[37]. T. Yang, et al. "Level measurement for saline with a small surface area using high frequency electromagnetic sensing technique," Measurement, vol. 101, pp. 118-125, 2017.

[38]. W. Zhou, M. Lu et al. "Three-dimensional electromagnetic mixing models for dual-phase steel microstructures," Applied Sciences, vol. 8, pp. 529, 2018.

[39]. M. Lu et al. "Prediction of the asymptotical magnetic polarization tensors for cylindrical samples using the boundary element method," 2015 IEEE Sensors Applications Symposium (SAS), pp. 1-4, 2015.

[40]. W. Yin et al. "An equivalent-effect phenomenon in eddy current nondestructive testing of thin structures," IEEE ACCESS, vol. 7, pp. 70296 - 70307, 2019.

[41]. M. Lu et al. "Measurement of ferromagnetic slabs permeability based on a novel planar triple-coil sensor," IEEE Sensors J., vol. 20, no. 6, pp. 2904-2910, 2020.

[42]. J.R.S. Avila, M. Lu et al. "Accurate measurements of plate thickness with variable lift-off using a combined inductive and capacitive sensor," NDT \& E International, vol. 110, pp. 102202, 2020. 
[43]. H. Xu, M. Lu et al. "Imaging Weld Cross-Section Using a Novel Frequency Feature in Multi-Frequency Eddy Current Testing," InsightNon-Destructive Testing and Condition Monitoring, vol. 61, pp. 738743, 2019

[44]. W. Yin et al. "Permeability invariance phenomenon and measurement of electrical conductivity for ferrite metallic plates," Insight-NonDestructive Testing and Condition Monitoring, vol. 61, pp. 472-479, 2019.

[45]. L. Yin et al. "Detection of corrosion pits based on an analytically optimised eddy current sensor," Insight-Non-Destructive Testing and Condition Monitoring, vol. 60, pp. 561-567, 2018.

[46]. M. Lu et al. "Determining the magnetic permeability of ferrite steel strip by a custom inversion method," Proc. 12th ECNDT, pp. 1-8, 2018.

[47]. J. Tang et al. "Bio-impedance spectroscopy for frozen-thaw of biosamples: Non-contact inductive measurement and finite element (FE) based cell modelling," Journal of Food Engineering, vol. 272, pp. 109784, 2020.

[48]. M. Lu, W. Zhu, L. Yin, A. J. Peyton, W. Yin, and Z. Qu, "Reducing the lift-off effect on permeability measurement for magnetic plates from multifrequency induction data," IEEE Transactions on Instrumentation and Measurement, vol. 67, no. 1, pp. 167-174, Jan. 2018.
[49]. M. Lu, L. Yin, A. J. Peyton and W. Yin, "A novel compensation algorithm for thickness measurement immune to lift-off variations using eddy current method," IEEE Transactions on Instrumentation and Measurement, vol. 65, no. 12, pp. 2773-2779, Dec. 2016.

[50]. M. Lu, X. Meng, W. Yin, Z. Qu, F. Wu, J. Tang, et al., "Thickness measurement of non-magnetic steel plates using a novel planar triplecoil sensor,” NDT \& E International, vol. 107, 2019. 